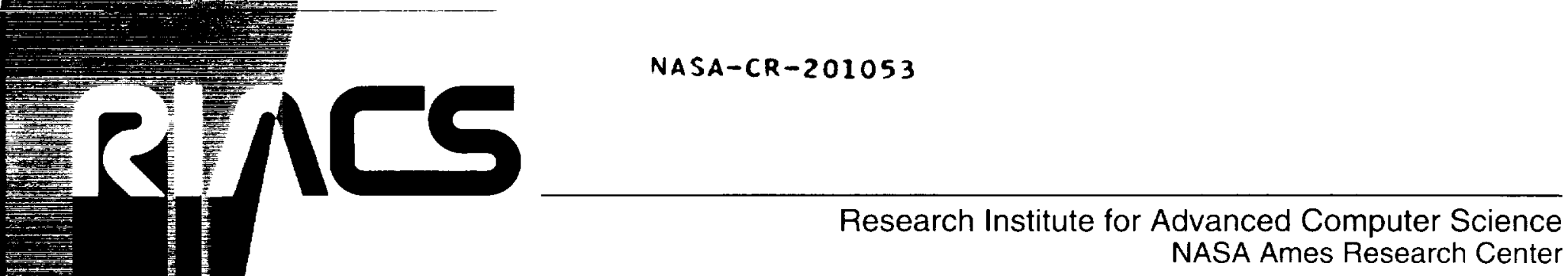

\title{
A Solution Adaptive Structured/Unstructured Overset Grid Flow Solver with Applications to Helicopter Rotor Flows
}

\author{
Earl P. N. Duque \\ Rupak Biswas \\ Roger C. Strawn
}

RIACS Technical Report 95.09

April 1995

Paper No. AIA A-95-1766, presented at the AIAA 13th Applied Aerodynamics Conference, San Diego, California, June 19-21, 1995 



\title{
A Solution Adaptive Structured/Unstructured Overset Grid Flow Solver with Applications to Helicopter Rotor Flows
}

\author{
Earl P. N. Duque \\ Rupak Biswas \\ Roger C. Strawn
}

The Research Institute of Advanced Computer Science is operated by Universities Space Research Association, The American City Building, Suite 212, Columbia, MD 21044, (410) 730-2656

Work reported herein was supported by NASA via Contract NAS 2-13721 between NASA and the Universities Space Research Association (USRA). Work was performed at the Research Institute for Advanced Computer Science (RIACS), NASA Ames Research Center, Moffett Field, CA 94035-1000. 



\title{
A SOLUTION ADAPTIVE STRUCTURED/UNSTRUCTURED OVERSET GRID FLOW SOLVER WITH APPLICATIONS TO HELICOPTER ROTOR FLOWS
}

\author{
Earl P.N. Duque \\ US Army Aeroflightdynamics Directorate, ATCOM, NASA Ames Research Center, Moffett Field, CA \\ Rupak Biswas \\ RIACS, NASA Ames Research Center, Moffett Field, CA \\ Roger C. Strawn \\ US Army Aeroflightdynamics Directorate, ATCOM, NASA Ames Research Center, Moffett Field, CA
}

\begin{abstract}
This paper summarizes a method that solves both the three dimensional thin-layer Navier-Stokes equations and the Euler equations using overset structured and solution adaptive unstructured grids with applications to helicopter rotor flowfields. The overset structured grids use an implicit finite-difference method to solve the thin-layer Navier-Stokes/Euler equations while the unstructured grid uses an explicit finite-volume method to solve the Euler equations. Solutions on a helicopter rotor in hover show the ability to accurately convect the rotor wake. However, isotropic subdivision of the tetrahedral mesh rapidly increases the overall problem size.
\end{abstract}

\section{Introduction}

A helicopter rotor blade generates a vortical wake that has a large effect on its performance, vibrations and noise. In order to accurately predict the performance of the rotor system, a numerical method must capture this important feature. In the past, numerical methods have relied upon empirically based models to approximate the wake effect (references [1] - [7]). More recently, researchers have used various CFD methods such as vorticity confinement coupled with full potential [8] and Euler or Navier-Stokes with periodic boundary conditions ([9] and [10]) in attempts to compute the wake structure from first principles.

All the methods mentioned above use single-block, body-conforming structured grids to discretize the entire flow field. Topological restrictions in single-block grids tend to degrade their efficient use in simulations of helicopter rotors. For example, single-block grids tend to have excessive and poorly placed grid points that increase required computer time, in-core memory and

This paper is declared a work of the U.S. Government and is not subject to copyright protection in the United States.

Presented at the 13th AIAA Applied Aerodynamics Conference, San Diego, CA, June 19-21, 1995. computer costs. Also, it is extremely difficult, if not impossible, to employ single-block grid schemes for rotor-body interactions where the relative motion between aircraft components is important.

In response to these disadvantages, two methodologies have emerged. One method, by Barth [11] and modified for rotary wing flows by Strawn and Barth [12], uses unstructured adaptive grids and an explicit time-integration finite-volume scheme. This method has the ability to readily refine the grid around flow structures as demonstrated by Strawn et. al. [13]. However, insufficient computer resources, inherent grid generation difficulties and solution accuracy within shear layers all tend to complicate large three-dimensional viscous flowfield computations.

Duque and Srinivasan [14] and Meakin [15] use overset structured grids for rotor computations. The overset grids allow for well formed grids at the rotor surface and separate grids to carry the wakes. These structured grid methods use implicit solution methods that allow for larger time steps compared to explicit methods. However, current structured grid methods are not well suited for three dimensional grid adaption for vortex wakes and cannot adequately adapt to an unsteady flowfield.

In contrast, dynamic unsteady remeshing and grid adaptation on unstructured grids has been presented by Lohner [16]. The ability to freely remove and add points within regions of interest is a major advantage of unstructured grids. In the wake of a helicopter blade, the solution method needs to resolve the convecting tip vortex and the vortex sheet. Unstructured grid methods work well within these flow regions. In addition, grids in this region do not require to be as fine as in viscous boundary layer regions near body surfaces. Therefore, the CFL limit of an explicit method does not constrain the time step.

In this paper, the advantages of structured and unstructured schemes are combined into a hybrid method that solves both the three dimensional thin-layer Navier-Stokes equations and Euler equations using overset structured and unstructured grids. The overset 
structured grids use the methodology developed by Srinivasan, et. al. [10]. The structured grids are overset onto an unstructured grid. The unstructured grid solution uses the method developed by Barth [11]. The unstructured grid adapts to the solution using the successive edge refinement and coarsening method of Biswas and Strawn [17].

The main objective of this paper is to determine grid adaption requirements for general helicopter wakes. A helicopter wake is unsteady and can interact with the oncoming blades. It consists of a tip vortex, root vortex and a vortical wake sheet. The paper presents a helicopter rotor in hover as a model problem and uses a number of different indicators to determine where to adapt the unstructured grid. An attempt is made to find an optimal indicator to identify helicopter wake regions. Results show that the current mesh adaption scheme can successfully refine the grid in rotor wake regions. However, isotropic subdivision of the tetrahedral elements tends to significantly increase the computer costs.

\section{Methodology}

The structured grid flow solver is that by Srinivasan, et. al. [10]. This method solves the thin-layer NavierStokes equations shown in Equation 1.

$$
\frac{\partial}{\partial t} \hat{Q}+\frac{\partial}{\partial \xi} \hat{E}+\frac{\partial}{\partial \eta} \hat{F}+\frac{\partial}{\partial \zeta} \hat{G}=R e^{-1} \frac{\partial}{\partial \zeta} \hat{S}
$$

Reference [10] describes the finite-difference implicit numerical scheme used for the solution of Equation 1. Briefly, the numerical algorithm spatially differences the flux terms using a Roe upwind-biased scheme for all three coordinate directions with higherorder MUSCL-type limiting to model shocks accurately. The method advances the solution in time using the LUSGS (lower-upper symmetric Gauss-Seidel) implicit scheme. Currently the scheme is third-order accurate in space and first-order accurate in time. The solver assumes fully turbulent flow for the blades and uses the algebraic turbulence model of Baldwin and Lomax [18] to estimate the eddy viscosity.

The unstructured grid flow solver is the code developed by Barth [11] and later modified by Strawn and Barth [12] for rotary wing applications. This program solves the finite-volume form of the Euler equations for rotary wing aerodynamics, shown in Equation 2, where $u$ is the vector of primitive variables, density, velocity and pressure, $F$ is the flux vector, $\Omega$ is the control volume and $\partial \Omega$ is its surface area.

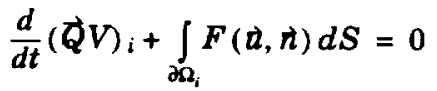

Special boundary conditions enforce the hovering rotor solution in inertial space coordinates. For a rotor in hover, the grid domain encompasses an appropriate fraction of the rotor azimuth. The unstructured grid flow solver then enforces the periodicity by forming control volumes that include the information from opposite sides of the domain. A no-slip boundary condition is used on the blade surface.

At the far field boundaries, the flow field magnitudes and direction depend upon the predicted rotor thrust as described by Srinivasan, et. al. [10] and Strawn and Barth [12]. This field is a linear superposition of momentum and potential theory. Momentum theory states that at some computed thrust and at some region far away from the rotor plane, mass flows out at twice the induced velocity at the rotor plane and over half its area. With potential theory, the rotor acts like a potential sink with the mass flux into the domain distributed over some far field area. This combination of boundary conditions yields a steady-state solution to the otherwise unsteady rotor in hover problem.

With overset grids, a sequence of grid sub-domains overlap. At the appropriate outer and resulting interior boundaries, the conserved flow data are interpolated between the separate grids. Consider, for example, the simple three grid system in Figure 1, where grids 1 and 2 are structured grids and grid 3 is an unstructured grid. Furthermore, grid 1 creates a hole within grid 2 and grid 2 creates a hole in grid 3. Grid 1 interpolates outer boundary data from grid 2 . Grid 2 interpolates outer boundary from grid 3 and hole data from grid 1 . Grid 3 obtains hole boundary data from grid 2 .

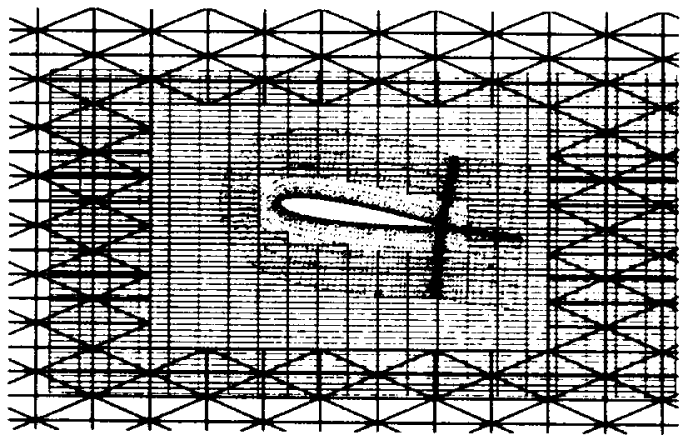

Figure 1. Three Grid Example Case

The current method uses the edge subdivision methodology developed by Biswas and Strawn [17],[19] to adapt the unstructured grid to the solution. The adaption scheme is applied in successive steps that either refine or coarsen tetrahedral elements according edge-based adaption indicator. The adaption method targets a userspecified number of edges for coarsening or refinement.

The adaption indicator is a scalar function that depends upon an appropriate combination of geometric 
and flow field information. This paper uses vorticity magnitude as the general adaption indicator. However, the vorticity magnitude is highly nonlinear with very high levels at the tip vortex and wake sheet. Scaling the vorticity by the edge length between two nodes as shown in Equation 3 can alleviate excessive grid clustering at these high gradient regions.

$$
\text { indicator }=\frac{\left(\omega_{j 2}+\omega_{j 1}\right) L_{e d g e}}{2}
$$

After each successive refinement step, the flow solver updates the flow field data and further converges the solution. However, the computed vorticity magnitude in the adapted region may further increase, causing repeated subdivision near the vortex and in the wake sheet. Excessive refinement can cause poor distribution of grid points which results in an inefficient use of available computer memory. Constraining the levels of refinement helps to more evenly distribute the grid refinement. In the current work, the grids are allowed to refine up to a maximum of three subdivision levels.

The helicopter rotor wake is the predominant flow feature in hover. Further geometric constraints were imposed to help improve the tip vortex resolution. The final adaption indicator uses a scaled vorticity that allows the grid to refine to only three levels. The adaption is then limited to occur only within 2 chords of the blade tip radius.

The rotor solutions in this paper use the structured/ unstructured overset grid system reported earlier by Duque [20]. This grid system models the rectangular planform two bladed rotor by Caradonna and Tung [21]. This blade has a NACA0012 cross section, a squared off tip and an aspect ratio of 6 . The overset grid system consists of four separate grids - one grid for the main rotor blade, two grids for the root and the tip and one for the far field unstructured wake grid. As shown in Figure 2, the blade grid captures the overall shape of the geometry under investigation. The grid accurately resolves the blade tips through the tip cap grid illustrated in Figure 2. These grids are overset onto an unstructured grid that is generated by algebraically subdividing an existing structured grid into tetrahedral elements.

The rotor in hover computations were performed at one of the subsonic tip hover test condition reported by Caradonna and Tung [21] $\left(\mathrm{M}_{\text {tip }}=0.439, \mathrm{Re}=1.83 \mathrm{mil}-\right.$ lion, $8^{\circ}$ Collective). The solutions reported earlier with this grid system were used as initial solutions to the adaption cases. Three different adaption indicators (scaled vorticity, level constraint and geometric constraint) were applied. Through adaptive refinement, the grids were brought to a maximum upper limit in the total number of elements. This upper limit gives a good comparison of the effect of each adaption indicator for a given amount of computational resources.

\section{Results and Discussion}

In the following calculations, the unstructured grid was limited to a maximum of 1.3 million tetrahedral elements, 1.5 million edges, or 230,000 nodes. A maximum of seven refinement/coarsening steps was implemented. Each grid refinement/coarsening step required approximately $300 \mathrm{CPU}$ seconds and a maximum of $96 \mathrm{MW}$ of memory on a Cray C-90. Between each refinement step, the unstructured-grid flow solver was run for 250 iterations or 2400 single CPU seconds to converge the solution. The combined structured/ unstructured flow solver required a maximum of $32 \mathrm{MW}$ of in-core memory on a Cray C-90.

The discussion gives a progressive comparison of the adapted grids along different cross sections of the unstructured grid. The results show that one must carefully choose and apply the adaption indicator for efficient use of available computational resources. All of the indicators based on vorticity demonstrate the ability to adapt to the tip vortex, root vortex, and wake sheet. However, the relative resolution of these flow features is highly dependent on the specifics of the adaption indicator. We assume that the most important flow features in the rotor wake are the tip vortices.

One problem with tetrahedral meshes is that it is very difficult to subdivide the grid in a truly anisotropic manner. Isotropic subdivision rapidly leads to high computational costs and the results will show that tetrahedral meshes may not be the ideal unstructured grid element for helicopter wakes. This is because the predominate features in helicopter wakes are tip vortices, which are inherently anisotropic. Hexahedral, or mixedelement meshes are much better suited for anisotropic subdivision than their tetrahedral counterparts but these were not used in the present study.

Figure 3 illustrates the grid for the initial and each of the adapted grids at the periodic plane $90^{\circ}$ behind the blade trailing edge. For comparison, Figure 3 a shows the initial coarse grid. Figure $3 b$ shows the final grid obtained using the scaled vorticity grid indicator. The grid shows refinement at the tip vortex region as denoted. In this region, the grid shows multiple levels of grid refinement. Grid refinement at the root vortex location is also seen.

Figure 3c shows the grid at the same location but with a maximum limit of three refinement levels. Four flow features become evident - initial tip vortex, wake sheet, root vortex and first passage of tip vortex. The major differences between this adapted grid and the previous are the increased adaption on the wake sheet and 
the multiple levels of adaption on the root vortex. The first vortex passage also shows more adaption levels than with the previous indicator.

Figure 3d shows the periodic plane grid with vorticity scaling, subdivision level limits and with adaption constrained to two chords inboard and outboard of the tip. The first noticeable difference between this case and the previous two is the lack of adaption at the root vortex. This indicator also yields more edge refinement surrounding the primary tip vortex region. No refinement occurs in the wake sheet region. The refinement in the first vortex passage location shows a larger refinement area.

We now move closer to the blade and take a planar cut through the unstructured grid. The cut is parallel to and 1.2 chords behind the blade trailing edge. Figure $4 \mathrm{a}$ illustrates the initial coarse grid. The scaled vorticity indicator result, Figure $4 \mathrm{~b}$, shows fine grid refinement at the tip vortex. This grid cut also shows evidence of wake roll up and some refinement in the region of the tip vortex first pass.

Figure $4 c$ shows the grid when limited to three subdivision adaption levels. This constraint allows the grid to adapt on more of the inboard wake sheet and on the first vortex blade passage. The tip vortex and the inboard wake sheet show the maximum three levels of adaption from the tip to the root. The first vortex passage shows two levels of refinement. The region just below the first passage shows evidence of refinement for the second blade passage.

Adding the location constraint helps to push more refinement from the inboard wake sheet to the tip vortex, Figure 4d. As shown, refinement on the inboard wake sheet cuts off rather abruptly. The three levels of refinement is maintained at the initial tip vortex formation. The first blade passage now has a larger refined region as compared to the two previous indicators. The second blade passage also shows more refinement in its respective region.

As we move closer to the blade trailing edge, the grid is further refined on the tip vortex and wake sheet. As the refinement increases, the level of vorticity magnitude that convects with the unstructured grid also increases. The unstructured grid then tends to refine to the level of the gradients in the flow field. The smallest gradient scales for a Navier-Stokes computation is the boundary layer height. Although the unstructured grid could theoretically refine to the fine scales, the isotropic subdivision requirement quickly increases the computer memory requirement.

The grid interface boundary between the structured and unstructured overset grid highlights this problem with the isotropic subdivision requirement. Figure 5a shows the initial interface boundary. The grid then refines via the scaled vorticity, Figure $5 b$. Many refinement levels allows for many points to lie within the tip vortex region. At the wake sheet, the grid begins to approach the boundary layer scales. Note that far inboard, the grid abruptly coarsens. This change occurs because the scheme distributes most of its available resources into the elements towards the tip. For a fixed number of total elements, the adaption onto the fine wake sheet and tip vortex scales has absorbed a large number of the total allowed elements.

Figure 5c shows the interface boundary with a maximum of three refinement levels. The grid shows a more distributed refinement in comparison to the previous case. The grid refines along the entire blade span. The root vortex, tip vortex and wake sheet all show three grid refinement levels. As seen in the wake cut and periodic plane, the level-constrained adaption had more refinement in the wake sheet and tip vortex regions. This indicator prevents excessive refinement in the wake sheet and allows the available resources to be applied more evenly.

Figure 5d shows the effect of geometrically limiting the refinement. The adapted region is similar to the previous case but it doesn't allow refinement on the inboard blade sections. As seen earlier, this indicator allows for the refinement to occur predominately in the tip vortex and allows the solution to carry the tip vortex further and with less dissipation.

The tip vortex location and strength has a large effect on the blade loads, especially in hover. Therefore, the adaption applied here should help to improve the prediction of blade loads. There is little difference between the loads predicted using the three different adaption indicators. Figure 6 compares blade loads obtained by the initial unadapted grid solution, the adapted grid solution and the measured loads. All three were obtained by blade surface pressure integration. The adapted solution shows an improved load distribution at the blade tip region.

The solution adaptive grid has a large impact on the predicted flow field. The increased resolution in the tip vortex allows the solver to convect the wake further and improves wake convection for up to two blade passages. Figure $7 \mathrm{a}$ illustrates the wake via a surface of constant vorticity. The surface shows the overall features of the three dimensional tip vortex and wake sheet. The wake sheet rolls up into the tip vortex and the surface holds its shape for half a blade revolution. The tip vortex begins to drop off as shown by the reduction of the surface. Figure $7 \mathrm{~b}$ further illustrates the wake structure through vorticity magnitude contours. The contours are shown at the two periodic planes and on a plane parallel to the blade feather axis. The contours are clipped at a minimum level of 0.01 to a maximum level of 0.1 . At these 
levels, the maximum vorticity has dropped off by an order of magnitude in comparison to the maximum vorticity levels at the overset boundary. The contours show the dissipation of the tip vortex and wake. The cut plane shows two distinct tip vortices, although they are much reduced in strength.

\section{Conclusion and Future Work}

This paper presents a new method capable of computing the viscous regions of helicopter blades and adapting the grid to the resulting wake solutions. The method performs the computations by solving the equations of motion upon a system of overset structured grids and solution adaptive unstructured grids. Various implementations of adaption indicators show that appropriate constraints on grid refinement help to properly distribute the grid points. However, the results also show that unstructured tetrahedral elements are not well suited for helicopter rotor flowfields. The tip vortex is the most important flow feature which is inherently an anisotropic feature. In future work, elements that allow anisotropic subdivision such as hexahedral elements will be used.

\section{References}

[1] Caradonna, F.X., Desopper, A., and Tung, C., "Finite Difference Modeling of Rotor Flows Including Wake Effects", Paper \#2.7, Eighth European Rotorcraft Forum, Aix-en Provence, France, August 1982.

[2] Strawn, R.C., and Caradonna, F.X., "Conservative Full-Potential Model for Unsteady Transonic Rotor Flows", AIAA Journal, Vol.25, No. 2, February 1987, pp. 193-198.

[3] Chang, I-C., and Tung, C., "Numerical Solution of the Full-Potential Equation for Rotor and Oblique Wings using a New Wake Model.", AIAA Paper 85-0268, January 1985.

[4] Chen, C.L., McCroskey, W.J., and Ying, S.X., "Euler Solution of Multibladed Rotor Flow", Vertica, Vol. 12, No. 3, 1988, pp.303-313.

[5] Agarwal, R.K., and Deese, J.E., "Navier-Stokes Calculations of the Flow field of a Helicopter Rotor in Hover", AIAA Paper 88-0106, 1988.

[6] Srinivasan, G.R., and McCroskey, W.J., "NavierStokes Calculations of Hovering Rotor Flowfields“, Journal of Aircraft, vol. 25, No. 10, October 1988, pp. 865-874.

[7] Wake, B.E., and Sankar, N.L., "Solutions of the Navier Stokes Equations for the Flow about a Rotor Blade ", Journal of the American Helicop- ter Society, Volume 34, Number 2, April 1989. pp.13-23.

[8] Ramachandran, K., Tung, C. and Caradonna, F.X., "Rotor Hover Performance Prediction Using a Free Wake, CFD Method", Journal of Aircraft, pp. 1105-1110, Vol. 26, No. 12, December 1989

[9] Chen, C.L., McCroskey, W.J. and Obayashi, S., "Numerical Simulation of Helicopter MultiBladed Flows", AIAA Paper 88-0046, Jan. 1988.

[10] Srinivasan, G.R., Obayashi, S., Baeder, J.D., and McCroskey, W.J., "Flow field of a Lifting Hovering Rotor - A Navier-Stokes Simulation", Proceedings of the 16th European Rotorcraft Forum, pp. 3.5.1 - 3.5.16, Vol. 1, September 1990.

[11] Barth, T., "Aspects of Unstructured Grids and Finite-Volume Solvers for the Euler and NavierStokes Equations", Unstructured Grid Methods for Advection Dominated Flows, AGARD Report 787, May 1992.

[12] Strawn, R.C. and Barth, T., "Helicopter Rotor Flows using Unstructured Grid Scheme ", American Helicopter Society Journal, Volume 38, Number 2, April 1993, pp. 61-67.

[13] Strawn, R.C., Garceau, M. and Biswas, R., "Unstructured Adaptive Mesh Computations of Rotorcraft High-Speed Impulsive Noise", 15th AIAA Aeroacoustics Conference, Long Beach, CA, October 1993, AIAA Paper 93-4359.

[14] Duque, E.P.N. and Srinivasan, G.R., "Numerical Simulation of a Hovering Rotor using Embedded Grids", Proceedings of the 48th AHS Annual Forum and Technology Display, Washington, D.C., June 3-5, 1992.

[15] Meakin, R., "Moving Body Overset Grid Methods for Complete Aircraft Tiltrotor Simulations," 11th AIAA Computational Fluid Dynamics Conference, Orlando, Florida, AIAA Paper 93-3350, July 1993.

[16] Lohner, R., "Finite Element Methods in CFD: Grid Generation, Adaptivity and Parallelization", AGARD Report 787, Unstructured Grid Methods for Advection Dominated Flows, May 1992.

[17] Biswas, R., and Strawn, R.C., "A New Procedure for Dynamic Adaption of Three-Dimensional Unstructured Grids,", Applied Numerical Mathematics, Vol 13, No. 6, pp. 437-452, February 1994.

[18] Baldwin, B.S. and Lomax, H., "Thin-Layer Approximation and Algebraic Model for Separated Turbulent Flows", AIAA 16th Aerospace Sciences Meeting, Huntsville, AL, January 1618, 1978. 
[19] Biswas, R., and Strawn, R.C., "Dynamic Mesh Adaption for Tetrahedral Grids," Presented at the 14th International Conference on Numerical Methods in Fluid Dynamics, Bangalore, India, July 11-15, 1994.

[20] Duque, E.P.N., “A Structured/Unstructured Embedded Grid Solver for Helicopter Rotor Flows", AHS 50th Annual Forum, Washington D.C., May 11-13, 1994.
[21] Caradonna, F.X. and Tung, C., "Experimental and Analytical Studies of a Model Helicopter Rotor in Hover", NASA TM-81232, September 1981.

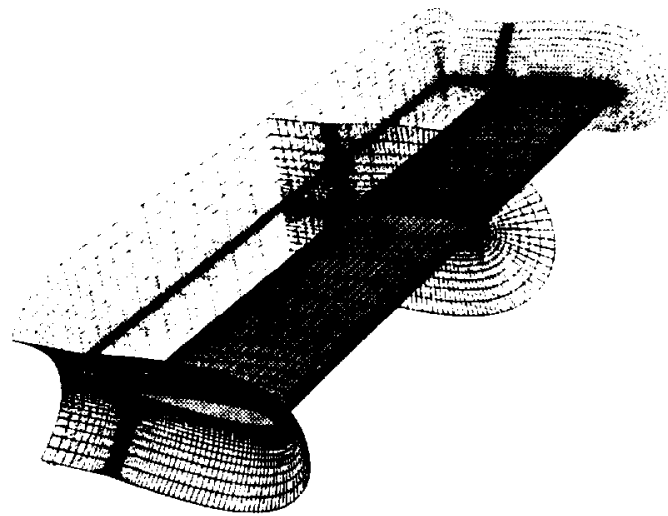

Blade Grid

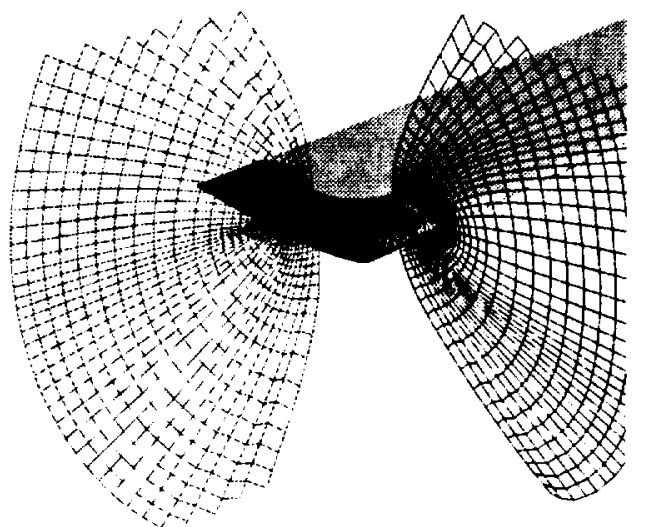

Tip Cap and Root Cap Grid

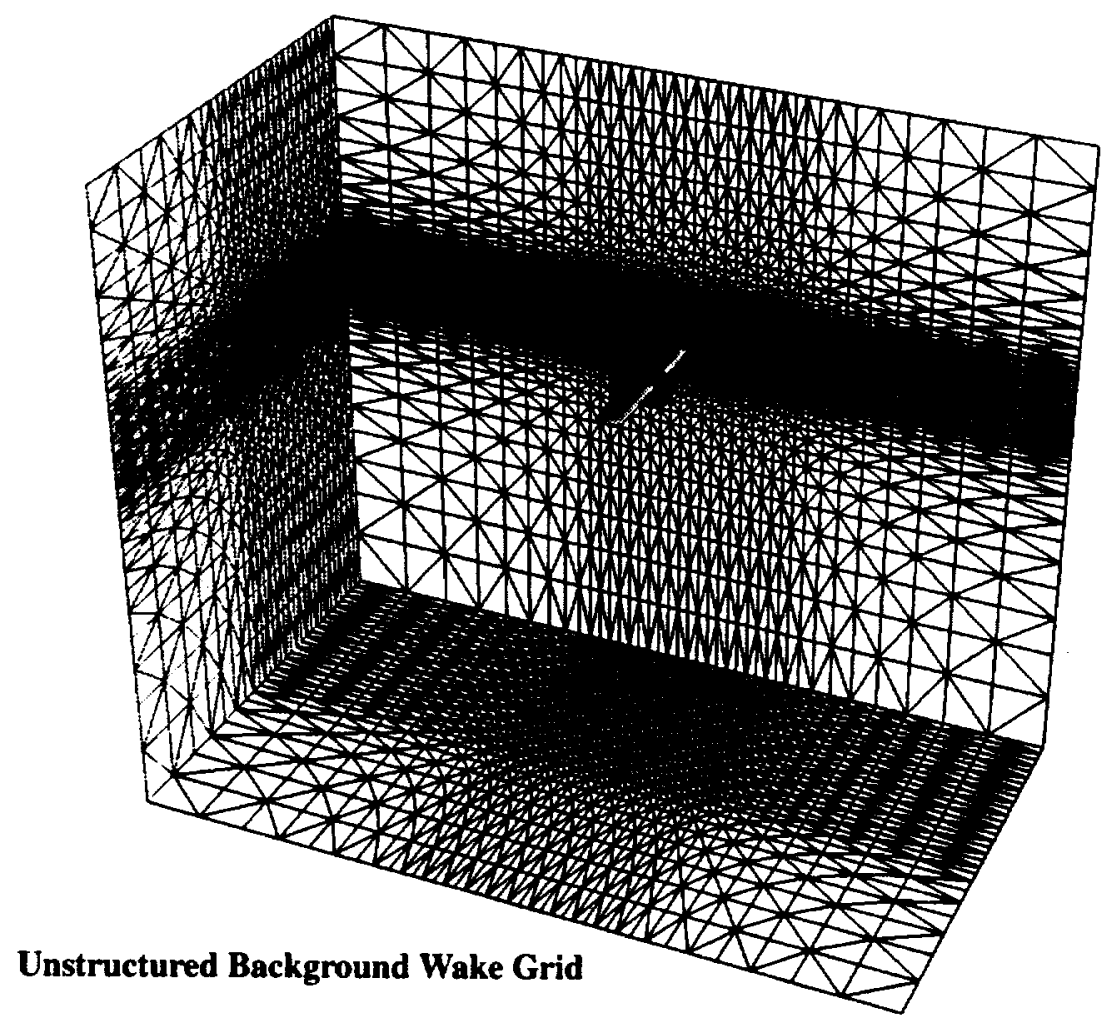

Figure 2: Rotor Grid Systems

6

American Institute of Aeronautics and Astronautics 


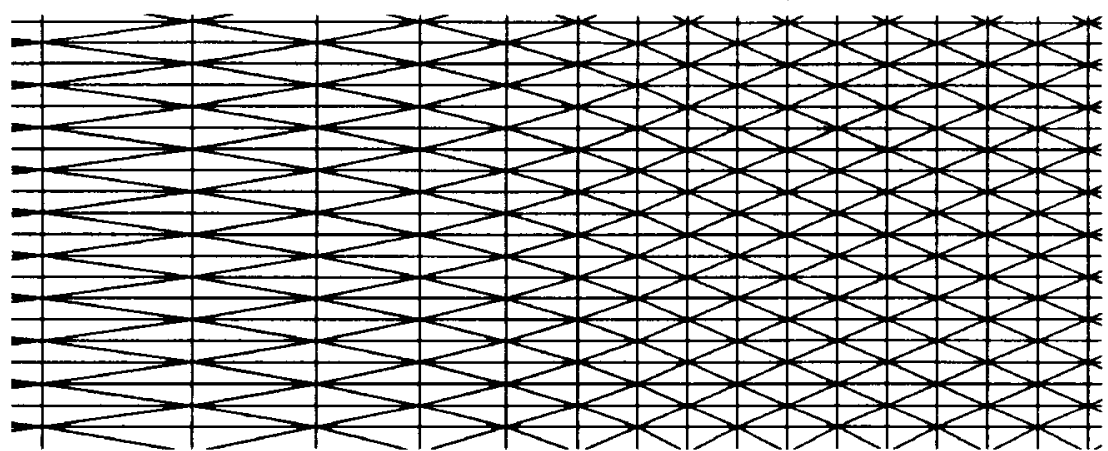

a) Initial Grid

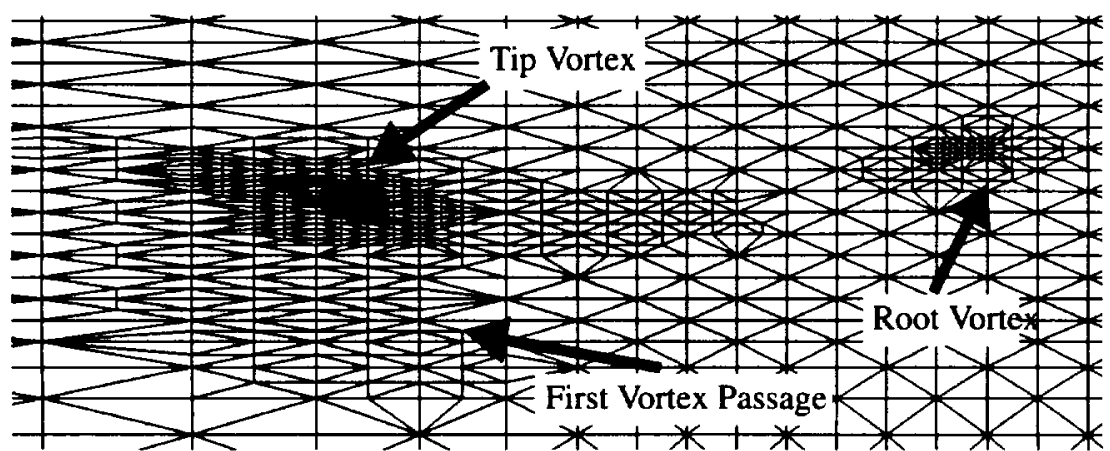

b) Scaled Vorticity

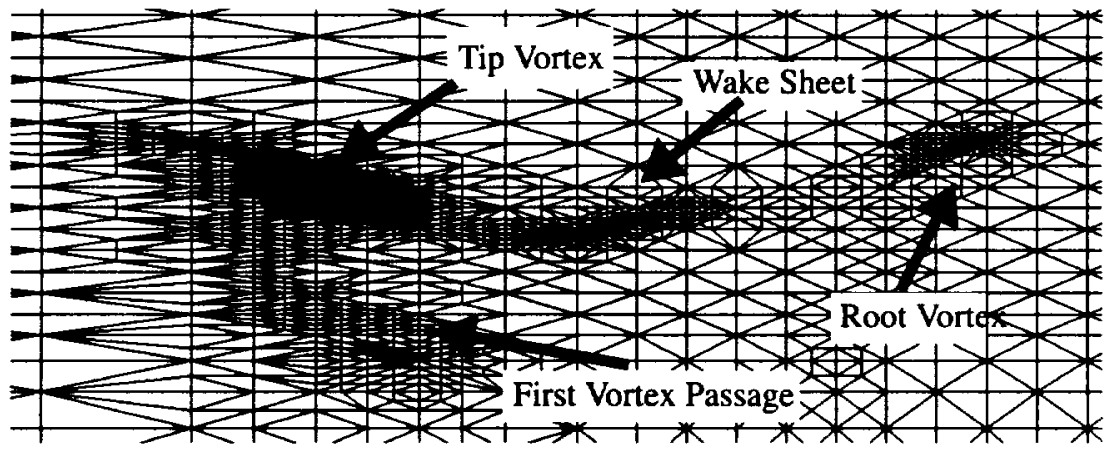

c) Level Constrained

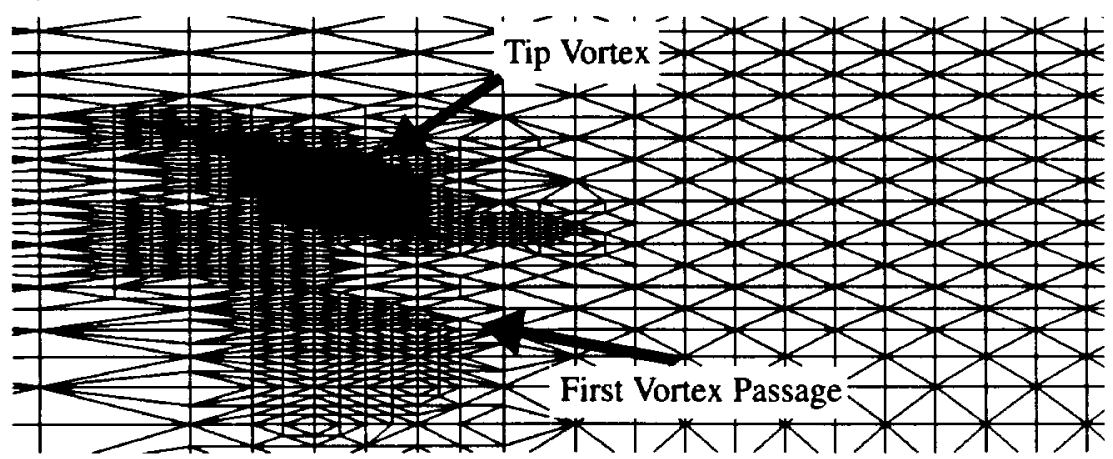

d) Level and Location Limited

Figure 3. Unstructured grid at Periodic Plane 


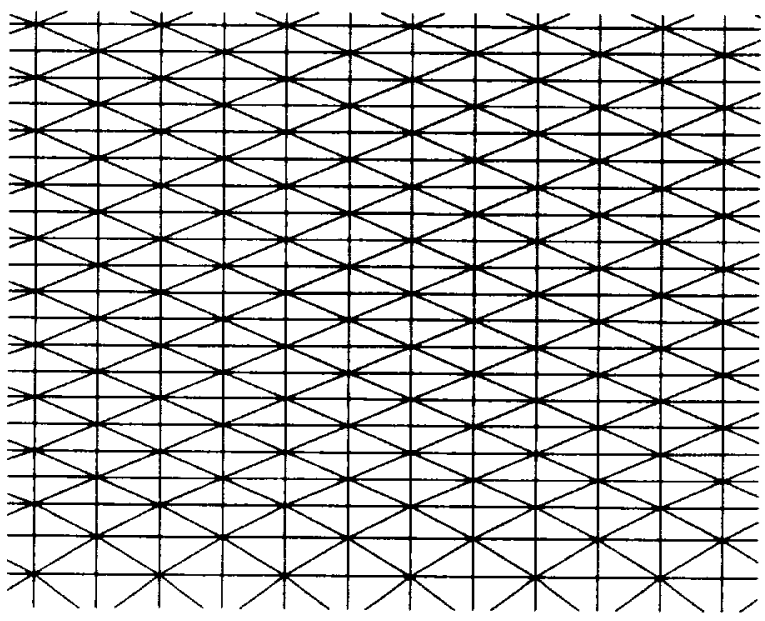

a) Initial

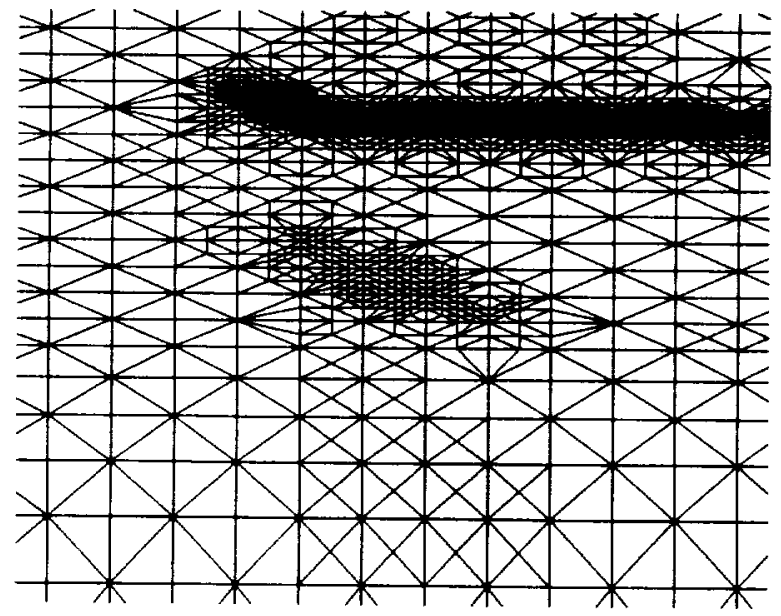

c) Level Constrained

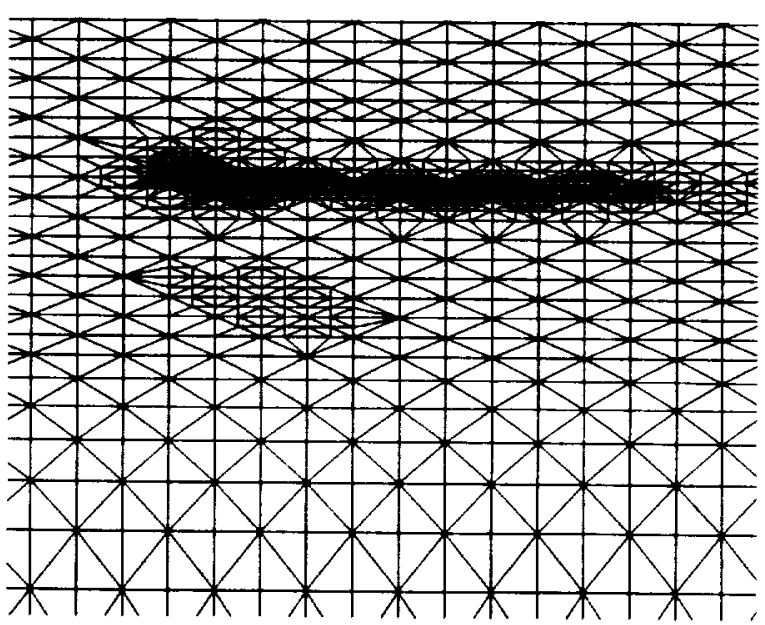

b) Scaled Vorticity

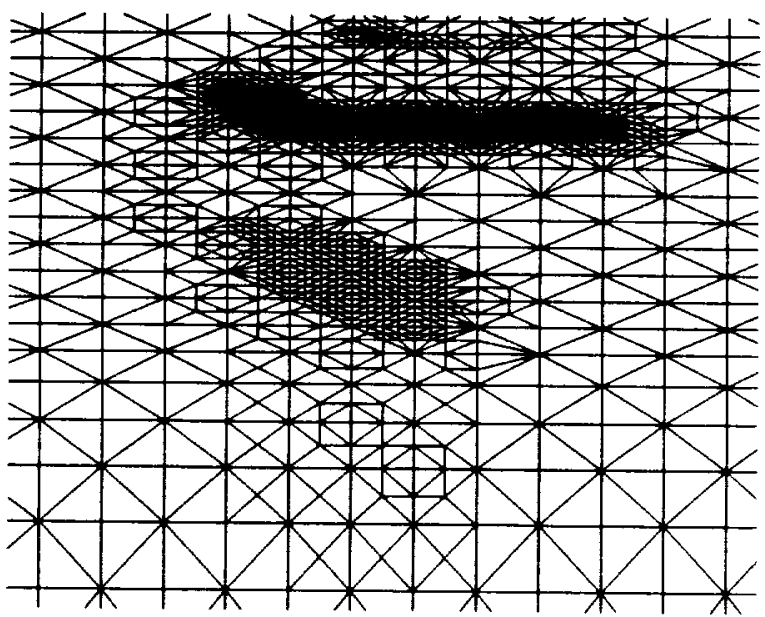

d) Level and Location Constrained

Figure 4. Unstructured grid at 1.2 Chords Behind Trailing Edge 


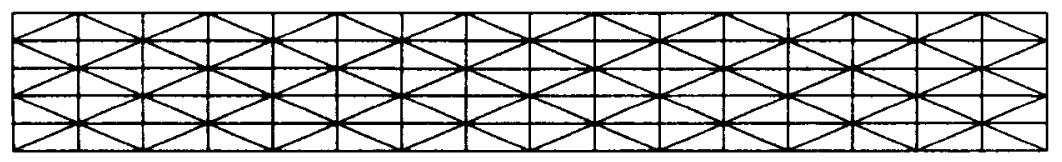

a) Initial

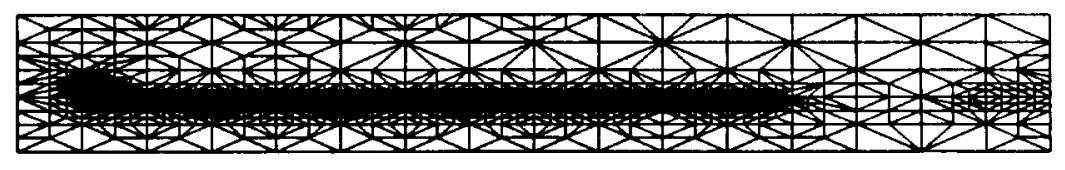

b) Scaled Vorticity

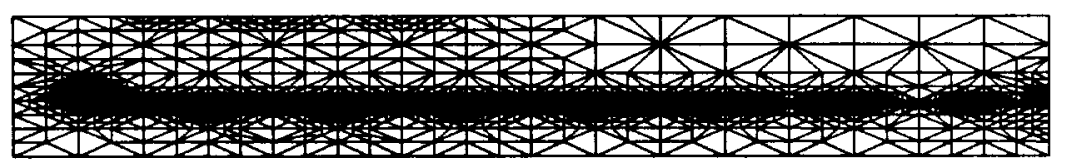

c) Level Constrained

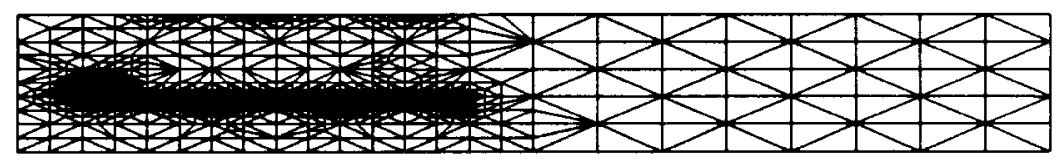

d) Level and Location Constrained

Figure 5. Structured/Unstructured Grid Interface Boundarye

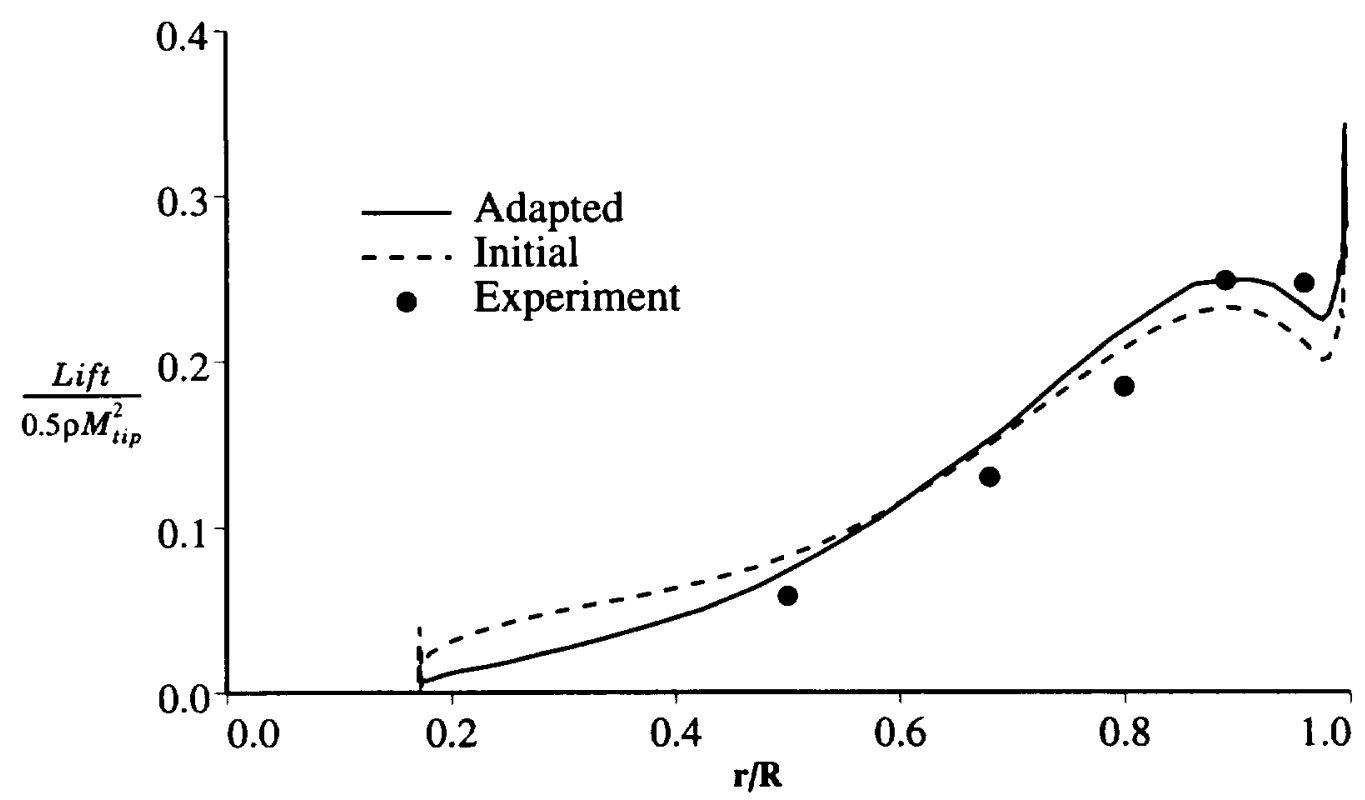

Figure 6. Spanwise Loading with Adapted and Initial Unstructured Wake Grid 


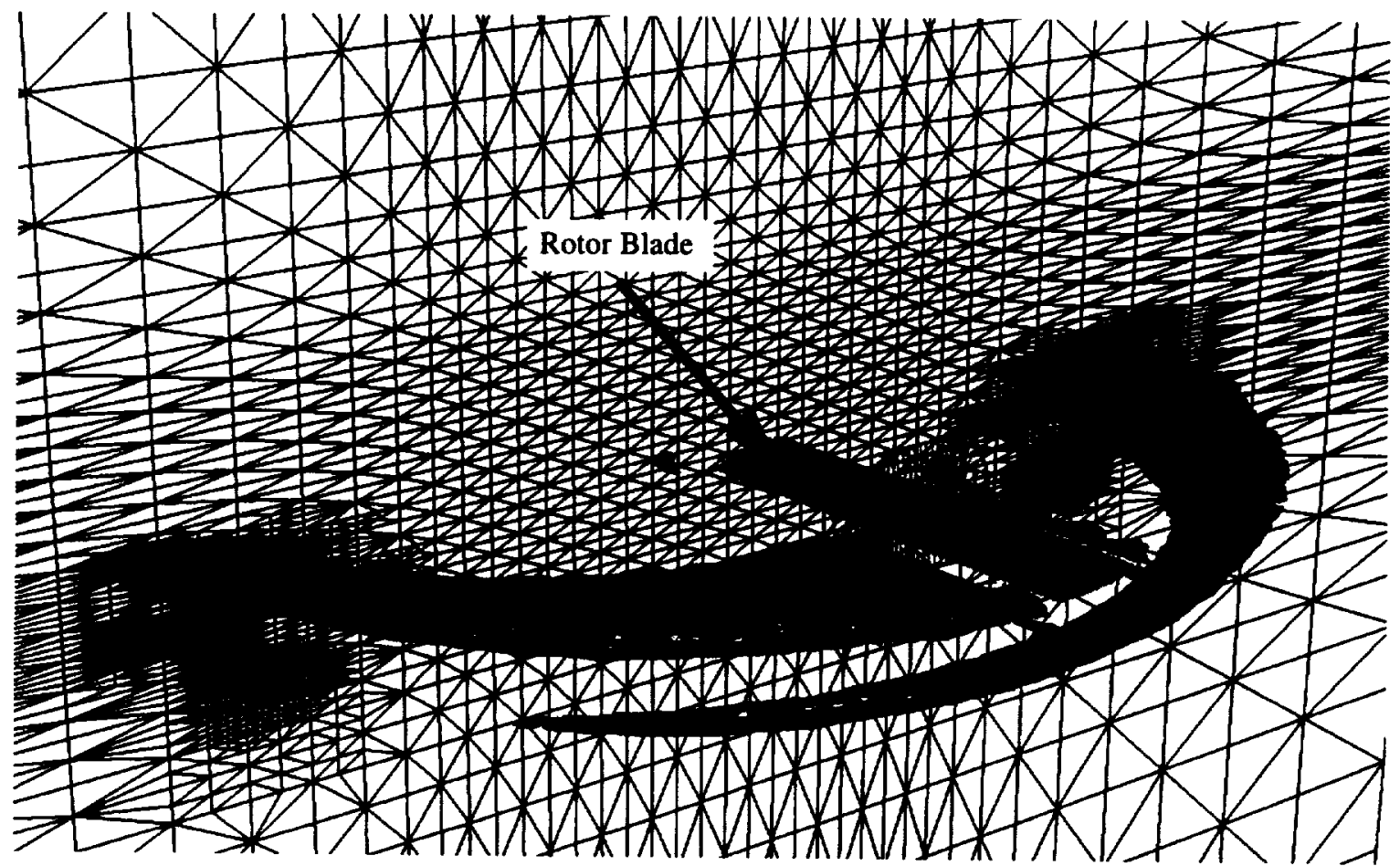

a) Isosurface of Voricity $=0.03$

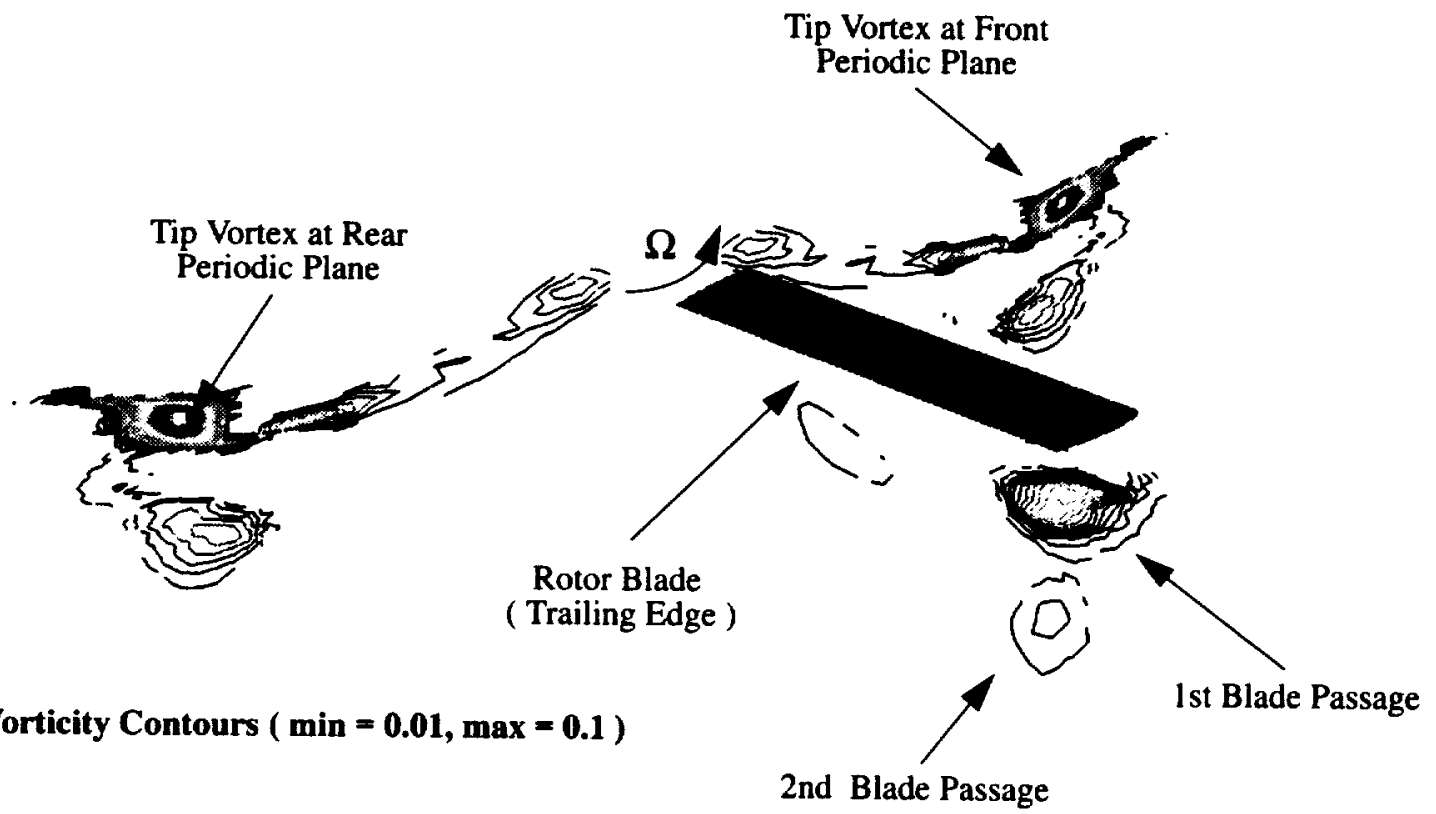

Figure 7. Isosurface of Vorticity and Vorticity Contours Level and Geometry Limited Unstructured Adapted Grid 

